\title{
On Bohr's response to the clock-in-the-box thought experiment of Einstein*
}

\author{
V Hnizdo \\ National Institute for Occupational Safety and Health, 1095 Willowdale Road, Morgantown, WV \\ 26505, USA
}

\begin{abstract}
The recent analysis of De la Torre, Daleo and García-Mata of the reply of Bohr to the famous clock-in-the-box challenge of Einstein is criticized.
\end{abstract}

\begin{abstract}
Recently, De la Torre, Daleo and García-Mata (TDG) [1] have subjected to a 'careful and irreverent' analysis the arguments of Einstein and Bohr in their dialog concerning the famous clock-in-the-box gedanken experiment devised by Einstein in an attempt to disprove the uncertainty relation for energy and time [2], and reached the conclusion that 'Einstein's argument is flawed while Bohr's reply is wrong.' In the present note, we would like to point out that it is the TDG analysis of the balancing procedure assumed in Bohr's reply that is flawed, and to uphold Bohr's simple inequality characterizing this balancing as correct - if not 'obviously', then on a little reflection.
\end{abstract}

The inequality in question,

$$
\Delta p<T g \Delta m
$$

puts an upper bound on the uncertainty $\Delta p$ of the momentum of a box whose mass has been determined to an uncertainty $\Delta m$ using a spring balance in a balancing procedure taking a time $T ; g$ is here the acceleration due to gravity. The rest of this paragraph is an elaboration on Bohr's terse justification of this inequality. Weighing on a spring balance, as on any balance, is essentially a position measurement (but also the preparation of a suitable momentum state, a point to which we shall return below) in which the position of a pointer attached to the mass being weighed is determined against a scale fixed firmly to the reference frame. The balancing procedure of TDG, in agreement with that implied by Bohr, consists of adding to or removing from the box progressively smaller masses $\Delta m$ until the pointer coincides with a reference mark on the scale to within some latitude $\Delta q$. Now, according to the uncertainty principle, the uncertainty $\Delta p$ in the momentum state of the box then will be of the order of $\hbar / \Delta q$, but this uncertainty has an upper bound due to the fact that the driving force of the final balancing stage is the weight $\Delta m g$ of the last mass

\footnotetext{
*This work was conducted independently of official duties in the Centers for Disease Control and Prevention (CDC), and the views do not necessarily represent those of the CDC.
} 
increment (or decrement) $\Delta m$, and thus it cannot impart to the box an impulse greater than $\Delta m g T_{\Delta m}$, where $T_{\Delta m}$ is the time that the final stage takes to complete. If this is not seen as 'obvious', a simple classical energy-balance calculation bears it out immediately. Consider a mass $m$ suspended in the state of rest from a spring of spring constant $k$, and suppose that a mass $\Delta m$ is added to it. Assuming for the moment no damping, the sum of the elastic, gravitational and kinetic energies at a distance $q$, measured vertically down from the original equilibrium position, remains equal to the initial zero total energy:

$$
\frac{1}{2} k q^{2}-\Delta m g q+\frac{1}{2}(m+\Delta m) v^{2}=0 .
$$

Here, only the gravitational energy of the increment $\Delta m$ need be considered, as the weight of the original mass $m$ is balanced by the spring force at the original equilibrium position $q=0$. The substitution in (2) of the value $q=\Delta m g / k$, which is where a spring force $-k q$ balances the weight $\Delta m g$ of the mass increment and the system reaches the maximum speed $v=v_{\max }$, yields for the maximum momentum $p_{\max }$ the value

$$
p_{\max }=(m+\Delta m) v_{\max }=\Delta m g \sqrt{\frac{m+\Delta m}{k}}=\Delta m g \frac{\tau}{2 \pi}
$$

where $\tau$ is the oscillation period of the system. Now, it takes at least a time $T_{\Delta m} \sim \tau$ to bring the system to the state of approximate rest at the new equilibrium position by reducing suitably its oscillations, and so we have indeed

$$
\Delta p \lesssim p_{\max }=\Delta m g \frac{\tau}{2 \pi}<\Delta m g T_{\Delta m}
$$

As $T_{\Delta m}<T$, Bohr's inequality (1), where $T$ is the time available for the whole balancing procedure, will be thus satisfied to an ample margin.

Contrary to what TDG assert, damping of oscillations leading to dissipation of the kinetic energy of the box can be allowed. In the weighing of the box before the release of a 'photon', a transfer of energy to the box is not critical. In the weighing after the release, however, it is permissible to transfer only an energy much smaller than $\Delta m c^{2}$ to the internal energy of the box. A complete damping of the oscillations dissipates an energy $K_{\Delta m}=\frac{1}{2} p_{\max }^{2} /(m+\Delta m)$, which, according to equation (3), equals $(\Delta m g)^{2} / 2 k$, and so the condition $K_{\Delta m} \ll \Delta m c^{2}$ can be written in terms of the new equilibrium position $q=\Delta m g / k$ as $q \ll 2 c^{2} / g \sim 10^{16} \mathrm{~m}-$ and surely that would be satisfied more than amply in any meaningful weighing procedure, gedanken or 'real'. In any case, the transfer of heat energy to the box can be prevented by using as the damping mechanism the friction between the spring, suitably shaped for this purpose, and a medium, and conducting the generated heat energy from the spring to the environment of the whole system. Oscillations can be reduced also by adding the given mass increment $\Delta m$ to the box gradually - for example, by breaking $\Delta m$ into suitably small parts and adding these to the box one by one in a suitable time sequence (classically, oscillations are eliminated when a mass increment $\Delta m \ll m$ is brought onto the box linearly in a time equal to the period $\tau=2 \pi(m / k)^{1 / 2}$, see [3]).

The above considerations stem from the realization that the completion of a proper weighing procedure amounts to the preparation of a state of approximate rest in which momentum as well as position must be suitably determined. This important point seems to 
be overlooked in most criticisms of Bohr's response, but it has been emphasized by Peierls, who treats the weighing procedure essentially as a momentum measurement [4].

The upper bound (1) on the momentum uncertainty, together with the uncertainty relation $\Delta p \Delta q \sim \hbar$, means that the uncertainty $\Delta q$ in the box's position has a lower bound,

$$
\Delta q>\frac{\hbar}{T g \Delta m}
$$

Bohr comments this by saying [2]: 'The greater the accuracy of the reading $q$ of the pointer, the longer must, consequently, be the balancing interval $T$, if a given accuracy $\Delta m$ of the weighing of the box with its content shall be obtained.' This should not be misunderstood as meaning that the increase of the balancing time $T$ brought about by just a decrease in the damping of the oscillations would permit a smaller uncertainty $\Delta q$, since it is the freeoscillation period $\tau$, and that is controlled by the spring constant $k$, that determines the maximum possible momentum uncertainty $\Delta p \sim \hbar / \Delta q$ for a given value of $\Delta m$ (see equation (4)). Bohr used inequality (5) and general relativity's 'red-shift' formula $\Delta T=T g \Delta q / c^{2}$ to confirm the energy-time uncertainty relation $\Delta E \Delta T>\hbar$ for the uncertainty $\Delta E=\Delta m c^{2}$ in the energy of a 'photon' and the uncertainty $\Delta T$ in the timing of its release from the box by a momentary opening of a shutter operated by a clock in the box. TDG claim that $\Delta T$ is in Bohr's usage the indeterminacy in the balancing time $T$ of the box, despite Bohr's statement [2] that the clock 'will change its rate in such a way that its reading in the course of a time interval $T$ will differ by an amount $\Delta T$.' This clearly means that the timing of the opening of the shutter operated by the clock will be uncertain to within the amount $\Delta T$ because the latter is, according to the 'red-shift' formula, directly proportional to the displacement of the clock from the reference mark with respect to which the clock was synchronized with the laboratory time,"] and that displacement is uncontrollable within the latitude $\Delta q$. Thus Bohr's meaning of $\Delta T$ is the same as that assigned to it by Einstein, contrary to what TDG assert.

TDG attempt to refute (1) by a counterexample in which the box is described by a harmonic-oscillator coherent state; in such states the momentum uncertainty is fixed at $\Delta p=\left(\frac{1}{2} \hbar m \omega\right)^{1 / 2}$ and the energy uncertainty is given by $\Delta E=\hbar \omega|\alpha|$, where $\omega$ and $\alpha$ are the oscillator frequency and an arbitrary complex parameter, respectively. Putting $\Delta E=$ $\Delta m c^{2}$, and choosing $|\alpha|<\left(c^{2} / T g\right)(m / 2 \hbar \omega)^{1 / 2}$, where $T$ is now an arbitrary time parameter, TDG obtain immediately a momentum uncertainty $\Delta p>T g \Delta m$, which would contradict (1). However, such reasoning is flawed on at least two accounts. First, it identifies the uncertainty in the mass of the box itself with that of the oscillator's total bound-state energy $E$, which is the sum of the kinetic and elastic potential energies of the box. Second, it ignores the actual time needed to bring the box into the desired state in which its position remains within the given latitude $\Delta q \sim \hbar / \Delta p$ on the reference mark of the fixed laboratory scale. Inequality (1) simply says that a time $T>\Delta p / g \Delta m$ is needed for the preparation of such a state in a balancing procedure that involves a force $\Delta m g$, and nothing in the argument of TDG disproves the validity of this statement. Thus if the TDG identification $\Delta m=\hbar \omega|\alpha| / c^{2}$

\footnotetext{
1 The distinction between the internal time of the clock in the box and the external laboratory time is important here, see [5].
} 
were correct, the balancing procedure resulting in a coherent harmonic-oscillator state with the desired properties would require a time $T>\Delta p / g \Delta m=\left(c^{2} /|\alpha| g\right)(m / 2 \hbar \omega)^{1 / 2}$ —and that directly contradicts the TDG choice of the value of the parameter $|\alpha|$.

The time involved in the preparation of a state is of importance here, not the magnitude of the state's momentum spread relative to the spread in total energy. One can find easily quantum states in which the momentum spread $\Delta p$ is greater by any given suitably dimensioned factor than the spread $\Delta E$ in the total energy of the state, a trivial example being any stationary bound state, in which, by definition, $\Delta E=0$, but $\Delta p \neq 0$. For the TDG argument, the most favourable value of the parameter $\alpha$ is $\alpha=0$, which gives the stationary ground state of a harmonic oscillator. Suppose, for the sake of the argument, that the box is prepared in such a state and that the readings $q$ on the balance scale are calibrated in terms of the mass $m$ as $k q=m g$. Then, despite the vanishing total-energy-uncertainty $\Delta E$ in the given state, the uncertainty $\Delta m$ to which the box's mass is determined is nonzero, satisfying the relation

$$
\Delta m g \approx k \Delta q=\frac{k \Delta p}{m \omega}=\frac{2 \pi}{\tau} \Delta p
$$

where we used the relation $\Delta q=\Delta p / m \omega$ between the position and momentum uncertainties in a stationary state of a harmonic oscillator of frequency $\omega=2 \pi / \tau=(\mathrm{k} / \mathrm{m})^{1 / 2}$. Relation (6) was obtained without any consideration given to the time involved in the preparation of the quantum state concerned-but when the oscillator period $\tau$ is accepted as a lower bound on the time needed to prepare the state, relation (6) agrees with relation (4), which was obtained classically for a balancing procedure explicitly involving time. However, it should be noted here that the fact that the box is a macroscopic system means that its preparation in any pure quantum state, and the TDG counterexample is such a state, would face fundamental difficulties even if the preparation procedure was not restricted to the balancing procedure considered.

Before closing, we would like to make two additional remarks. The first one is on the emphasis of TDG on the need to distinguish between classical 'uncertainty', by which they mean 'the lack of precision in the knowledge of a value assigned to an observable due to apparatus or experimental limitations', and quantum 'indeterminacy', which 'denotes the impossibility of assigning precise values to the observables of a system as prescribed by quantum mechanics;' TDG reproach Bohr for mixing confusingly the two notions in his argumentation. In this connection, TDG point out that quantum 'indeterminacies' of observables are not related by the same function that relates the observables themselves. But quantum 'indeterminacies' are not different in this respect from experimental 'uncertainties'. To use the example of the energy $E$ and momentum $p$ of a free particle, where $E=p^{2} / 2 m$ : when the mean momentum $|\bar{p}| \gg \Delta p$, the 'indeterminacy' $\Delta E \approx|d E / d p|_{p=\bar{p}} \Delta p=(|\bar{p}| / m) \Delta p$, exactly as for an experimental 'uncertainty' $\delta E \approx(|p| / m) \delta p$. While it must be admitted that it is often necessary to make a distinction between 'uncertainty' and 'indeterminacy', these two concepts are closely related in the context of establishing the empirical meaning of the quantum-mechanical formalism, which is the context in which Bohr uses such terms. According to Bohr, quantum mechanics would not be a satisfactory physical theory if it were not possible to demonstrate that there is harmony between what he called the possibilities of definition in the formalism on the one hand and the physical possibilities of measurement on the other [6] (the most elaborate embodiment of this point of view of Bohr, as 
applied to the case of quantum electrodynamics, is the famous analysis of the measurability of the electromagnetic field of Bohr and Rosenfeld [7]). There is such harmony only if one cannot prepare experimentally a state in which a physical quantity $Q$ has an 'uncertainty' $\delta Q$ while, according to quantum mechanics, that quantity can be defined in such a state only to an 'indeterminacy' $\Delta Q>\delta Q$, and, conversely, if the formalism does not allow a sharper definition of the values of physical quantities than that permitted by the measurement (albeit perhaps only gedanken) possibilities. Bohr believed that quantum mechanics is a satisfactory physical theory, and therefore that the 'uncertainty' $\delta Q$ of an experimental preparation must match the 'indeterminacy' $\Delta Q$ in all the physical quantities of a physical state; it is in this light that Bohr defended the uncertainty relations against the ingenious attempts of Einstein to disprove them. After Bohr's criticism of the clock-in-the-box thought experiment, Einstein abandoned his attempts at showing that simultaneous determination of two conjugate quantities is in principle experimentally possible, and instead began to argue 'only' for simultaneous reality of such quantities. The famous argument of Einstein, Podolsky and Rosen [8] that the quantum-mechanical formalism cannot provide a complete description of physical reality is constructed in this spirit.

The second remark concerns the point of TDG that Einstein's use of the photon as the particle with which he aimed to disprove the energy-time uncertainty relation was unfortunate, rendering his argument flawed. Indeed, already according to classical electrodynamics, an opening of the shutter for a short time $\Delta t$ would result in the emission of an electromagnetic-wave pulse containing frequencies in a range of width $\Delta \omega \sim 1 / \Delta t$, but this is a classical Fourier-analysis result that alone does not lead to quantum uncertainty relations (a classical electromagnetic pulse containing many frequencies still has a well-defined energy). Now, the photon, as a quantum particle, cannot be sharply localized in time and simultaneously have a sharply defined energy, just as a molecule, as a quantum particle, cannot be sharply localized in time and have a sharply defined kinetic energy. Nothing of substance would change in the clock-in-the-box experiment if Einstein used in it a gas of material particles like molecules instead of the electromagnetic radiation. If Essentially, the clock-in-the-box gedanken experiment boils down to the question whether it is possible, in a well-defined (though not necessarily realistic) measurement procedure, to determine arbitrarily accurately both a change in the energy of a system and the laboratory time when the change takes place, irrespective of the particular form the energy change assumesand that may be the emission of electromagnetic radiation, matter, or both. To argue, as TDG do, that Einstein assumed a non-existing particle, namely a well-localized photon of a well-defined energy, amounts to 'putting the cart before the horse', because while such a particle cannot be defined in the formalism of quantum theory, it is up to experiment (here, a gedanken one) to check whether that formalism is not too narrow for the scope of physical possibilities of measurement.

\footnotetext{
${ }^{2}$ This is why we prefer, following Peierls [4] and Pais [9], to employ the term 'clock' rather than 'photon' in the name we use for this gedanken experiment.
} 


\section{REFERENCES}

[1] De la Torre A C, Daleo A, and García-Mata I 2000 The photon-box Bohr-Einstein debate demythologized Eur. J. Phys. 21 253-260

[2] Bohr N 1949 Discussion with Einstein on epistemological problems in atomic physics Albert Einstein: Philosopher-Scientist ed P A Schilpp (La Salle: Open Court) pp 199241

Bohr N 1983 Quantum Theory and Measurement eds J A Wheeler and W H Zurek (Princeton, NJ: Princeton University Press) pp 9-49 (reprint of the original 1949 printing)

[3] Landau L D and Lifshitz E M 1976 Mechanics (London: Pergamon Press) sec 22, problem 2

[4] Peierls R 1979 Surprises in Theoretical Physics (Princeton, NJ: Princeton University Press) sec 1.8

[5] Aharonov Y and Reznik B 2000 "Weighing" a closed system and the time-energy uncertainty principle Phys. Rev. Lett. 84 1368-1370

[6] Aharanov Y and Petersen A 1971 Definability and measurability in quantum theory Quantum Theory and Beyond ed T Bastin (Cambridge: Cambridge University Press) pp $135-139$

[7] Bohr N and Rosenfeld L 1933 Zur Frage der Messbarkeit der elektromagnetischen Feldgrössen Mat. Phys. Medd. K. Dan. Vidensk. Selsk. 12 no 8

Bohr N and Rosenfeld L 1983 On the question of the measurability of electromagnetic field quantities Quantum Theory and Measurement eds J A Wheeler and W H Zurek (Princeton, NJ: Princeton University Press) pp 470-522 (Engl. transl.)

[8] Einstein A, Podolsky B, and Rosen N 1935 Can quantum-mechanical description of physical reality be considered complete? Phys. Rev. 47 777-780

[9] Pais A 1991 Niels Bohr's Times, In Physics, Philosophy, and Polity (Oxford: Clarendon Press) pp 427-428 